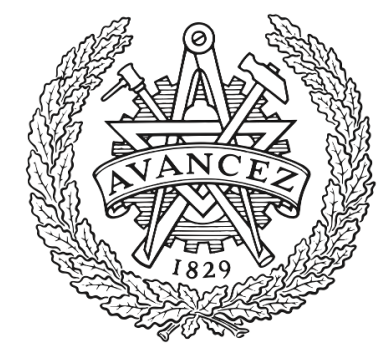

\title{
CHALMERS
}

UNIVERSITY OF TECHNOLOGY

\section{Selective coupling of superconducting charge qubits mediated by a tunable stripline cavity}

Downloaded from: https://research.chalmers.se, 2023-04-26 13:08 UTC

Citation for the original published paper (version of record):

Wallquist, M., Shumeiko, V., Wendin, G. (2006). Selective coupling of superconducting charge qubits mediated by a tunable stripline cavity. Physical Review B - Condensed Matter and Materials Physics, 74. http://dx.doi.org/10.1103/PhysRevB.74.224506

N.B. When citing this work, cite the original published paper. 


\title{
Selective coupling of superconducting charge qubits mediated by a tunable stripline cavity
}

\author{
M. Wallquist, V. S. Shumeiko, and G. Wendin \\ Chalmers University of Technology, SE-41296 Gothenburg, Sweden \\ (Received 9 August 2006; revised manuscript received 18 October 2006; published 15 December 2006)
}

\begin{abstract}
We theoretically investigate selective coupling of superconducting charge qubits mediated by a superconducting stripline cavity with a tunable resonance frequency. The frequency control is provided by a flux-biased $\mathrm{dc}$ superconducting quantum interference device attached to the cavity. Selective entanglement of the qubit states is achieved by sweeping the cavity frequency through the qubit-cavity resonances. The circuit is able to accommodate several qubits and allows one to keep the qubits at their optimal points with respect to decoherence during the whole operation. We derive an effective quantum Hamiltonian for the basic, two-qubit-cavity system, and analyze appropriate circuit parameters. We present a protocol for performing Bell inequality measurements, and discuss a composite pulse sequence generating a universal control-phase gate.
\end{abstract}

DOI: $10.1103 /$ PhysRevB.74.224506

PACS number(s): 85.25.Cp, 03.67.Lx

\section{INTRODUCTION}

Coherent coupling of superconducting qubits has been experimentally demonstrated for all major qubit types (charge, ${ }^{1,2}$ flux,${ }^{3-5}$ and phase ${ }^{6,7}$ qubits) using permanent direct qubit-qubit coupling, capacitive or inductive. A major challenge is to implement a tunable coupling of qubits required for any useful gate operation. Numerous suggestions in this direction have been discussed in recent literature together with related quantum gate protocols (for a review see, e.g., Ref. 8).

There are two conceptually different approaches to the tunable coupling. The first approach is to employ direct coupling schemes using Josephson junctions in the nonresonant regime as passive controllable elements, either capacitive ${ }^{9}$ or inductive. ${ }^{10-14}$ The second approach, which we adopt in this paper, suggests qubit coupling via a dynamic intermediate element, e.g., $L C$ oscillator or Josephson junction, which becomes entangled with a qubit during a two-qubit operation. In this scheme, the entanglement is achieved by tuning the qubit and the mediator in resonance, and then transferring the entanglement to another qubit by tuning the mediator and the second qubit in the resonance. Such coupling method has been first suggested ${ }^{15}$ and experimentally tested ${ }^{16}$ for the ion trap qubits. For superconducting qubits, qubit-oscillator entanglement has been demonstrated experimentally for a charge qubit coupled to a microwave stripline cavity, ${ }^{17}$ and a flux qubit coupled to a superconducting quantum interference device (SQUID) oscillator; ${ }^{18,19}$ the gate protocols based on controllable qubit-oscillator coupling have been theoretically discussed in Refs. 20 and 21.

The experimental setup with the qubit coupling to a distributed oscillator-stripline cavity ${ }^{17,22}$ possesses potential for scalability - several qubits can be coupled to the cavity. In this paper we investigate the possibility of using this setup for implementation of tunable qubit-qubit coupling and simple gate operations. Tunable qubit-cavity coupling is achieved by varying the cavity frequency by controlling magnetic flux through a dc SQUID attached to the cavity (see Fig. 1). An advantage of this method is the possibility of keeping the qubits at the optimal points with respect to decoherence during the whole two-qubit operation. The qubits coupled to the cavity must have different frequencies, and the cavity in the idle regime must be tuned away from resonance with all of the qubits. Selective addressing of a particular qubit is achieved by relatively slow passage through the resonance of a selected qubit, while other resonances are rapidly passed. The speed of the active resonant passage should be comparable to the qubit-cavity coupling frequency while the rapid passages should be fast on this scale, but slow on the scale of the cavity eigenfrequency in order to avoid cavity excitation. This strategy requires a narrow width of the qubit-cavity resonances compared to the differences in the qubit frequencies, determined by the available interval of the cavity frequency divided by the number of attached qubits. This consideration simultaneously imposes a limit on the maximum number of employed qubits. Denoting the difference in the qubit energies by $\Delta E_{J}$, the coupling energy by $\kappa$, the maximum variation of the cavity frequency by $\Delta \omega_{k}$, and the number of qubits by $N$, we summarize the above arguments with the relations $\kappa \ll \Delta E_{J}, N \sim \hbar \Delta \omega_{k} / \Delta E_{J}$. In the off-resonance state, the qubit-qubit coupling strength is smaller than the on-resonance coupling by the ratio $\kappa /\left(\hbar \omega_{k}-E_{J}\right) \ll 1$.

In the first part of the present paper, we analyze the quantum electrical circuit consisting of a superconducting stripline cavity, a dc SQUID, and single Cooper pair box (SCB) qubits, derive an effective quantum Hamiltonian for this circuit, and discuss the relevant circuit parameters.

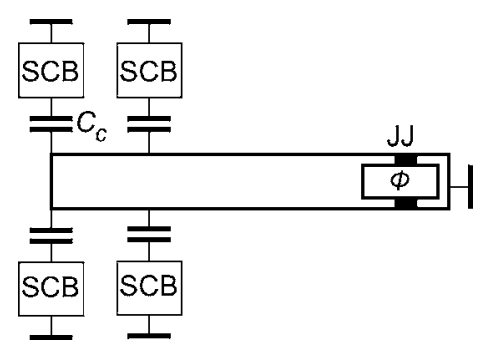

FIG. 1. Sketch of the device: charge qubits [single Cooper pair boxes (SCB)] coupled capacitively $\left(C_{c}\right)$ to a stripline cavity integrated with a dc SQUID formed by two large Josephson junctions $(\mathrm{JJ})$; cavity eigenfrequency is controlled by magnetic flux $\Phi$ through the SQUID. 


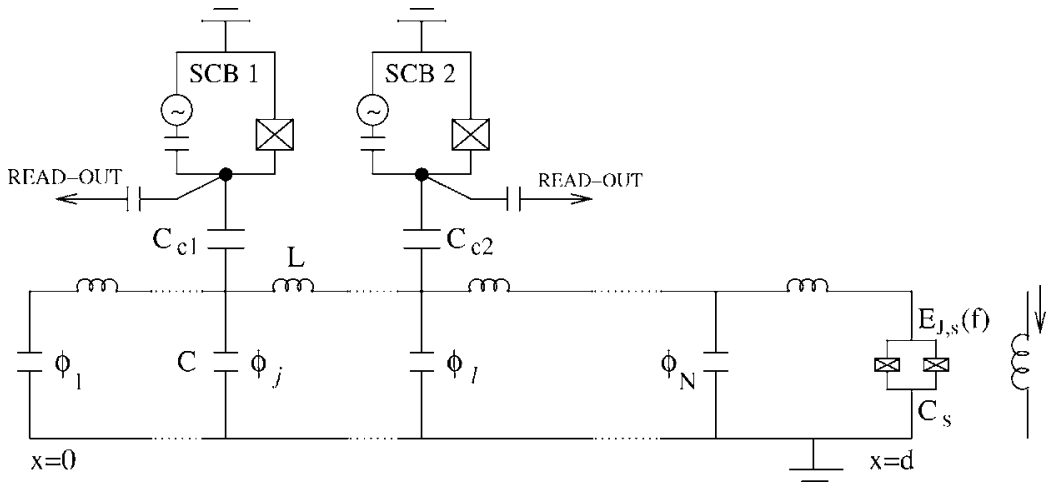

FIG. 2. Equivalent circuit for the device in Fig. 1: chain of $L C$ oscillators represents the stripline cavity, $\phi_{1}$ and $\phi_{N}$ are superconducting phase values at the ends of the cavity, $\phi_{j}$ and $\phi_{l}$ are local phase values where the qubits are attached; attached dc SQUID has effective fluxdependent Josephson energy $E_{J s}(f)$ and capacitance $C_{s}$; control line for tuning the SQUID is shown at the right; SCB qubits are coupled to the cavity via small capacitances $C_{c 1}$ and $C_{c 2}$.
Then, on the basis of the derived Hamiltonian, we discuss the Bell measurement protocol and a protocol for a conditional phase gate. We consider creating maximally entangled two-qubit states (Bell states) by sequentially sweeping the cavity through the resonances with the two qubits, ${ }^{20}$ and discuss the protocol for measuring the Clauser-Horne-ShimonyHolt $(\mathrm{CHSH})$ correlation function ${ }^{23}$ for such states, which is equivalent to testing the Bell inequality.

While considering the universal two-qubit gate, we take into account an important feature of our system - the linearity of the cavity, which does not allow implementation of the $\sqrt{\text { SWAP }}$ gate. ${ }^{21}$ We argue that the control-phase (CPHASE) gate is a genuine two-qubit gate for our system (cf. Ref. 20). We consider a protocol for this gate, which is much faster than the one suggested in Ref. 20, the present one being based on resonant rather than dispersive qubit-oscillator coupling. A major difficulty for constructing such a protocol is the generation of single- and two-photon states in the cavity (for the cavity initialized in the ground state); elimination of these auxiliary photon states requires a complex pulse sequence. ${ }^{24}$

In this paper, we explicitly discuss the coupling of charge qubits; however, the method of derivation of the effective quantum Hamiltonian also applies, with minor modifications, to the flux qubits, and the quantum protocols studied can be extended to this type of qubit system.

\section{CAVITY WITH VARIABLE FREQUENCY}

The resonant frequency of a one-dimensional stripline cavity depends on the boundary conditions. For example, if one end of the cavity is open while the other is connected to the ground, the spatial distribution of the superconducting phase along the cavity has a maximum at the open end and a node at the grounded end. This corresponds to the quarterwavelength resonator $d=\lambda / 4$, with the eigenmode wave vectors $k_{n}=(\pi / d)(n+1 / 2)$, where $d$ is the cavity length, the eigenmode frequencies being $\omega_{n}=(\pi v / d)(n+1 / 2)$, where $v$ is the velocity of the electromagnetic waves in the cavity. If, on the other hand, the second end is disconnected from the ground, the eigenmode wave vectors become $k_{n}=(\pi / d) n$, giving the frequencies $\omega_{n}=(\pi v / d) n$. The role of the $\mathrm{dc}$ SQUID attached to the stripline cavity in Fig. 1 is to vary the boundary condition at the right end: the first case (node) corresponds to a very large (formally infinite) Josephson energy of the SQUID, while the second case (antinode) corre- sponds to the fully suppressed Josephson energy. Thus, ideally, by changing the biasing magnetic flux through the SQUID by half a flux quantum, $0 \leq \Phi \leq \Phi_{0} / 2\left(\bmod \Phi_{0}\right)$, $\left(\Phi_{0}=h / 2 e\right)$, one should be able to sweep the eigenmode frequencies within the intervals $(\pi v / d) n \leq \omega_{n} \leq(\pi v / d)(n$ $+1 / 2$ ). In practice these intervals are narrower due to the finite maximum and nonzero minimum Josephson energies of the SQUID.

For a given eigenmode, the integrated stripline+SQUID system behaves as a lumped oscillator with variable frequency. Our goal in this section will be to derive an effective classical Lagrangian ${ }^{25,26}$ for this oscillator. To this end we consider in Fig. 2 an equivalent circuit for the device depicted in Fig. 1. A discrete chain of identical $L C$ oscillators, with phases $\phi_{i}$ across the chain capacitors $(i=1, \ldots, N)$, represents the stripline cavity; the dc SQUID is directly attached at the right end of the chain, while the superconducting Cooper pair boxes are attached via small coupling capacitors $C_{c 1}$ and $C_{c 2}$ to the chain nodes with local phases $\phi_{j}$ and $\phi_{l}$ (for simplicity we consider only two attached SCBs). The classical Lagrangian for this circuit,

$$
\mathcal{L}=\mathcal{L}_{S L}+\mathcal{L}_{\text {SQUID }}+\sum_{j=1,2}\left[\mathcal{L}_{q, j}+\mathcal{L}_{\text {coupl }, j}\right]
$$

consists of the stripline Lagrangian

$$
\begin{aligned}
\mathcal{L}_{S L}= & \sum_{i=1}^{N-1}\left(\frac{\hbar}{2 e}\right)^{2}\left(\frac{C \dot{\phi}_{i}^{2}}{2}-\frac{\left(\phi_{i+1}-\phi_{i}\right)^{2}}{2 L}\right)+\frac{\hbar^{2} C}{2(2 e)^{2}} \dot{\phi}_{N}^{2} \\
& -\frac{\hbar^{2}\left(\phi_{S, 1}-\phi_{N}\right)^{2}}{2(2 e)^{2} L},
\end{aligned}
$$

the SQUID Lagrangian,

$$
\mathcal{L}_{\text {SQUID }}=\sum_{i=1,2}\left(\frac{\hbar^{2}\left(C_{s} / 2\right)}{2(2 e)^{2}} \dot{\phi}_{s, i}^{2}+E_{J s, i} \cos \phi_{s, i}\right),
$$

the Lagrangians of the SCBs,

$$
\mathcal{L}_{q, j}=\frac{\hbar^{2} C_{j}}{2(2 e)^{2}} \dot{\phi}_{q, j}^{2}+\frac{\hbar^{2} C_{g}}{2(2 e)^{2}}\left(\dot{\phi}_{q, j}+\frac{2 e}{\hbar} V_{g, j}\right)^{2}+E_{J, j} \cos \phi_{q, j},
$$

and the capacitive SCB-stripline coupling, 


$$
\mathcal{L}_{\text {coupl }, j}=\frac{\hbar^{2} C_{c, j}}{2(2 e)^{2}}\left(\dot{\phi}_{j}+\dot{\phi}_{q, j}\right)^{2}
$$

The SQUID junction variables are related through the flux quantization relation $\phi_{s, 1}-\phi_{s, 2}=f$ to an externally applied magnetic flux $\Phi=\left(\Phi_{0} / 2 \pi\right) f=(\hbar / 2 e) f$, threading the SQUID ring. The self-inductance of the SQUID ring is assumed to be negligibly small compared to the Josephson inductances of the SQUID junctions. Then the SQUID can be described as a single junction with effective capacitance $C_{s}$ and fluxdependent Josephson energy

$$
E_{J s}(f)=\left[E_{J s, 1}^{2}+E_{J s, 2}^{2}+2 E_{J s, 1} E_{J s, 2} \cos (f)\right]^{1 / 2} .
$$

\section{A. Linear approximation}

Let us assume small amplitude of the plasma oscillation in the SQUID, $\phi_{s} \ll 1$, which implies the phase regime for the SQUID $E_{J s}(f) \gg(2 e)^{2} / 2 C_{s}$, and then adopt the harmonic oscillator approximation in Eq. (3),

$$
\mathcal{L}_{\text {SQUID }} \rightarrow \frac{\hbar^{2} C_{s}}{2(2 e)^{2}} \dot{\phi}_{s}^{2}-\frac{E_{J s}(f)}{2} \phi_{s}^{2},
$$

where $\phi_{s}=\left(\phi_{s, 1}+\phi_{s, 2}\right) / 2+\eta(f)$, and $\eta(f)$ is a constant phase shift, which can be neglected for adiabatic flux variations. The SQUID Josephson energy $E_{J s}(f)$, Eq. (6), reaches its maximum at zero magnetic flux, $E_{J s}^{m a x}=E_{J s, 1}+E_{J s, 2}$, while the minimum is approached at $f=\pi: E_{J s}^{\min }=\left|E_{J s, 1}-E_{J s, 2}\right|$ with $E_{J s}^{\min }>0$ due to the SQUID asymmetry.

To proceed to a continuum description of the stripline cavity, we introduce the distance $\Delta x$ between nodes $i$ and $i+1$, and express the stripline Lagrangian Eq. (2) in terms of the stripline capacitance and inductance per unit length,

$$
C_{0}=C / \Delta x, \quad L_{0}=L / \Delta x .
$$

Let $\Delta x$ go to zero and transform the node index $i$ into the continuous variable $x$. In the bulk of the cavity, the equation of motion of the field is a wave equation,

$$
\ddot{\phi}(x, t)-v^{2} \phi^{\prime \prime}(x, t)=0,
$$

where $v=1 / \sqrt{L_{0} C_{0}}$ is the wave velocity. It is convenient to express the wave velocity through the cavity inductance $L_{c a v}=d L_{0}$ and the cavity capacitance $C_{c a v}=d C_{0}$,

$$
v=\frac{d}{\sqrt{L_{c a v} C_{c a v}}} .
$$

The boundary condition at the cavity open end $(x=0)$,

$$
\phi^{\prime}(0, t)=0,
$$

requires that the only allowed solutions are of the form $\phi(x, t)=\phi_{1} \sin (k v t) \cos (k x)$. The boundary condition at the cavity right end $(x=d)$ reads

$$
\phi(d, t)=\phi_{s}(t)
$$

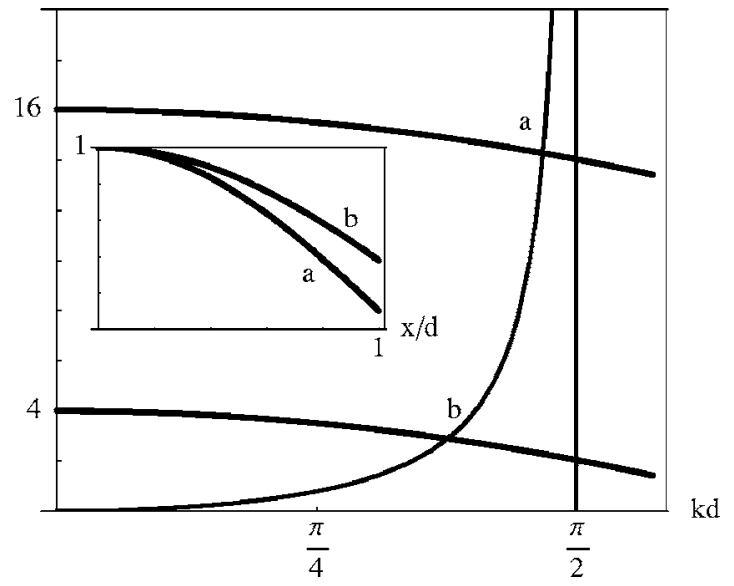

FIG. 3. Solution of dispersion equation (11) for first mode, $k d \leq \pi / 2(d \leq \lambda / 4)$, for large (a) and small (b) Josephson energies of the SQUID $\left[(2 e / \hbar)^{2} L_{c a v} E_{s}=16\right.$ and 4, respectively]; inset shows corresponding spatial distributions of the phase $\phi / \phi(0)$ in the cavity.

$$
\frac{\hbar^{2} C_{s}}{(2 e)^{2}} \ddot{\phi}(d, t)+\frac{\hbar^{2} d}{(2 e)^{2} L_{c a v}} \phi^{\prime}(d, t)+E_{J s}(f) \phi(d, t)=0 .
$$

A dispersion equation for the cavity eigenmodes results from Eq. (10) using the bulk solution to Eq. (8), and takes the form

$$
(k d) \tan (k d)=\frac{(2 e)^{2}}{\hbar^{2}} L_{c a v} E_{s}(f)-\frac{C_{s}}{C_{c a v}}(k d)^{2} .
$$

The solutions to this dispersion equation form an infinite set of eigenmodes with wavelengths $\lambda=2 \pi / k$ and frequencies $\omega_{k}=k v$.

The solutions to Eq. (11) are illustrated in Fig. 3: they are given by the intersection points of the function $k d \tan (k d)$ with the parabola, which is almost flat in the practically relevant limit, $C_{s} / C_{c a v} \ll 1$. The zeros of this function $(k d=n \pi, n=0,1, \ldots)$ correspond to an open right end of the cavity (disconnected SQUID), while the singular points $(k d=\pi / 2+\pi n)$ correspond to a closed cavity end (shortcircuited SQUID). These limits of the variation of the cavity wave eigenvectors, $n \pi \leq k_{n} d \leq \pi / 2+\pi n$, can be achieved when $E_{s}(f)$ varies between $\infty$ and 0 ; thus ideally the frequency can be tuned between $n \pi / \sqrt{L_{c a v} C_{c a v}}$ and $(\pi / 2+\pi n) / \sqrt{L_{c a v} C_{c a v}}$. In practice, the available frequency range is smaller, being limited by the value of the parameter $\left[(2 e)^{2} /\left(2 \hbar^{2}\right)\right] L_{c a v} E_{s}(0)$, which should be chosen large, and the minimum value of the SQUID Josephson energy $E_{s}(\pi)$ allowed by the SQUID asymmetry.

Let us return to the Lagrangian of the stripline cavity and the SQUID, Eqs. (2) and (7), and only consider a single eigenmode $\phi(x, t)=\phi_{1}(t) \cos (k x)$. In the continuum limit the Lagrangian will then take the form 


$$
\begin{aligned}
\mathcal{L}_{\text {osc }}= & \left(\frac{\hbar}{2 e}\right)^{2} \int_{0}^{d} d x\left(\frac{C_{0} \dot{\phi}_{1}^{2} \cos ^{2}(k x)}{2}-\frac{\phi_{1}^{2} k^{2} \sin ^{2}(k x)}{2 L_{0}}\right) \\
& +\frac{\hbar^{2} C_{s}}{2(2 e)^{2}} \dot{\phi}_{1}^{2} \cos ^{2}(k d)-\frac{E_{s}(f)}{2} \phi_{1}^{2} \cos ^{2}(k d) .
\end{aligned}
$$

After performing integration over $x$, and using the dispersion equation (11) we arrive at the effective $L C$ oscillator Lagrangian representing the integrated cavity+SQUID system,

$$
\mathcal{L}_{\text {osc }}=\frac{\hbar^{2} C_{k}}{2(2 e)^{2}} \dot{\phi}_{1}^{2}(t)-\frac{\hbar^{2}}{2(2 e)^{2} L_{k}} \phi_{1}^{2}(t) .
$$

The oscillator is described by the effective $k$-dependent capacitance $C_{k}$,

$$
C_{k}=\frac{C_{c a v}}{2}\left(1+\frac{\sin (2 k d)}{2 k d}\right)+C_{s} \cos ^{2}(k d),
$$

and the effective inductance $L_{k}$,

$$
\frac{1}{L_{k}}=\frac{(k d)^{2}}{2 L_{c a v}}\left(1+\frac{\sin (2 k d)}{2 k d}+\frac{2 C_{s}}{C_{c a v}} \cos ^{2}(k d)\right) .
$$

The frequency of the effective oscillator, defined in the usual way, $\omega_{k}=1 / \sqrt{C_{k} L_{k}}$, is equal to the frequency of the chosen cavity eigenmode as one should expect,

$$
\omega_{k}=\frac{k d}{\sqrt{L_{c a v} C_{c a v}}} .
$$

\section{B. Nonlinear correction}

While the stripline cavity alone is a linear electromagnetic system, attaching the dc-SQUID makes the integrated system nonlinear. Nonlinearity will introduce a nonequidistant correction to the quantized energy spectrum of the cavity, which may affect the gate protocols; in particular it is harmful for the conditional phase gate protocol considered later in the paper. Therefore it is important to estimate nonlinear effects produced by the SQUID on the cavity.

To this end we expand the SQUID potential in the boundary condition in Eq. (10), assuming $\phi_{s} \ll 1$, and keep a small cubic term,

$$
\begin{aligned}
& \frac{\hbar^{2} C_{s}}{(2 e)^{2}} \ddot{\phi}(d, t)+\frac{\hbar^{2} d}{(2 e)^{2} L_{c a v}} \phi^{\prime}(d, t)+E_{J s}(f)\left(\phi(d, t)-\frac{1}{6} \phi^{3}(d, t)\right) \\
& \quad=0 .
\end{aligned}
$$

The cubic term will introduce the third harmonic in the cavity,

$$
\phi(x, t)=\phi_{1} \sin (k v t) \cos (k x)+\phi_{3} \sin (3 k v t) \cos (3 k x),
$$

whose amplitude $\phi_{3}$ can be found from the boundary condition (17),

$$
\phi_{3}=-\frac{A_{k}}{24} \phi_{1}^{3}
$$

$$
A_{k}=\frac{\omega_{s}^{2} \cos ^{3}(k d)}{\left[\omega_{s}^{2}-9(k v)^{2}\right] \cos (3 k d)-\left(3 k d / L_{c a v} C_{s}\right) \sin (3 k d)} .
$$

Here we introduced the plasma frequency of the SQUID,

$$
\omega_{s}^{2}(f)=(2 e / \hbar)^{2}\left[E_{J s}(f) / C_{s}\right] .
$$

The cubic term also produces a shift of the resonance frequency given by the corrected dispersion equation

$$
\begin{aligned}
(k d) \tan (k d)= & \frac{(2 e)^{2}}{\hbar^{2}} L_{c a v} E_{s}(f)\left(1-\frac{1}{8} \omega_{s}^{2} \cos ^{2}(k d) \phi_{1}^{2}\right) \\
& -\frac{C_{s}}{C_{c a v}}(k d)^{2} .
\end{aligned}
$$

Taking the relation (11) into account, and omitting a small term $\sim C_{s} / C_{k} \ll 1$, we obtain the relative shift of the frequency

$$
\frac{\delta \omega_{k}}{\omega_{k}}=\frac{\delta k}{k}=-\frac{1}{2} B_{k} \phi_{1}^{2}, \quad B_{k}=\frac{(1 / 4) \cos ^{2}(k d)}{1+2 k d / \sin (2 k d)} .
$$

Such an amplitude-dependent frequency shift, on the other hand, can be recovered from the effective oscillator Lagrangian in Eq. (13) by adding the following nonquadratic term:

$$
\delta \mathcal{L}_{o s c}=\frac{\hbar^{2}}{2(2 e)^{2} L_{k}} B_{k} \phi_{1}^{4} .
$$

In the quantum regime, such a term will produce a deviation from the equidistant energy spectrum of the cavity. The magnitude of this deviation in the first perturbative order reads

$$
\delta E_{n}=-\frac{\hbar^{2}}{2(2 e)^{2} L_{k}} B_{k}\left\langle\phi_{1}^{4}\right\rangle_{n}=-\frac{6 n^{2}+6 n+3}{4} B_{k} E_{C k},
$$

where $E_{C k}=(2 e)^{2} / 2 C_{k}$ is the charging energy of the cavity, and $n$ is the energy level number. Thus we see that the nonlinear effect is proportional to the charging energy of the cavity. In order to neglect the nonlinear effect, this energy must be much smaller than the energy of the qubit coupling to the cavity (see below).

\section{QUBIT COUPLING TO THE CAVITY}

Now we take the SCB qubits in Eq. (1) into consideration, assuming that the coupling capacitances $C_{c, j}$ are small enough that the perturbation of the cavity eigenmodes due to the SCBs is negligible.

The cavity field $\phi_{j}$ at the point where $\mathrm{SCB} j$ is coupled is related to the effective oscillator variable $\phi_{1}$ by the relation $\phi_{j}=\phi_{1} \cos \left(k x_{j}\right)$, where $x_{j}$ is the position of qubit $j$ along the cavity. The coupling is described by the cross term in Eq. (5),

$$
\mathcal{L}_{i n t, j}=\frac{\hbar^{2}}{(2 e)^{2}} C_{c, j} \cos \left(k x_{j}\right) \dot{\phi}_{1} \dot{\phi}_{q, j} ;
$$

the quadratic terms in Eq. (5) give small renormalization of the qubit capacitance, $C_{\Sigma j}=C_{j}+C_{c, j}+C_{g}$, and the oscillator capacitance. 

form,

We transform the capacitive interaction into an inductive

$$
\mathcal{L}_{i n t, j}=\alpha_{j} E_{J, j} \phi_{1} \sin \phi_{q, j},
$$

using the transformation ${ }^{27}$

$$
\phi_{q, j} \rightarrow \phi_{q, j}+\alpha_{j} \phi_{1},
$$

with the coupling constant

$$
\alpha_{j}=C_{c, j} \cos \left(k x_{j}\right) / C_{\Sigma, j}, \quad \alpha_{j} \ll 1 .
$$

The SCB Lagrangian does not change during the transformation, whereas the oscillator undergoes displacement,

$$
\mathcal{L}_{L C}=\frac{\hbar^{2} C_{k}}{2(2 e)^{2}} \dot{\phi}_{1}^{2}-\frac{\hbar}{2 e} C_{g}\left(\alpha_{1} V_{g, 1}+\alpha_{2} V_{g, 2}\right) \dot{\phi}_{1}-\frac{\hbar^{2}}{2(2 e)^{2} L_{k}} \phi_{1}^{2},
$$

and small renormalization of the effective capacitance $C_{k} \rightarrow C_{k}-\sum_{j=1,2} C_{c, j} \cos \left(k x_{j}\right)\left[\cos \left(k x_{j}\right)-\alpha_{j}\right]$.

\section{A. Effective Hamiltonian}

We obtain the classical circuit Hamiltonian

$$
H=\sum_{j=1,2}\left[H_{j}+H_{i n t, j}\right]+H_{o s c}
$$

by introducing the conjugate momenta $n=(1 / \hbar) \partial L / \partial \dot{\phi}_{1}$ and $n_{j}=(1 / \hbar) \partial L / \partial \dot{\phi}_{q, j}$. Each SCB is described by the Hamiltonian

$$
H_{j}=E_{C, j}\left(n_{j}-n_{g, j}\right)^{2}-E_{J, j} \cos \phi_{q, j},
$$

where $E_{C, j}=(2 e)^{2} /\left(2 C_{\Sigma, j}\right)$ is the charging energy of the SCB island, and $n_{g, j}=C_{g} V_{g, j} /(2 e)$ is the (dimensionless) charge on the island induced by the gate voltage.

According to Eq. (29), the gate voltages also induce a charge $n_{0}=\alpha_{1} n_{g, 1}+\alpha_{2} n_{g, 2}$ on the oscillator. Because the oscillator charge is not quantized, this induced charge does not have any physical meaning, and can be eliminated using the gauge transformation $U^{\dagger} H_{o s c} U$ with $U=\exp \left(-i n_{0} \phi_{1}\right)$. The oscillator Hamiltonian then reads ${ }^{28}$

$$
H_{o s c}=E_{C k} n^{2}+E_{L k} \phi_{1}^{2},
$$

where $E_{C k}=(2 e)^{2} /\left(2 C_{k}\right)$ is the charging energy of the oscillator and $E_{L k}=\hbar^{2} /\left[2(2 e)^{2} L_{k}\right]$ its effective inductive energy. The interaction term in Eq. (30) is given by the expression in Eq. (26) with the opposite sign, $H_{i n t, j}=-\mathcal{L}_{i n t, j}$.

The Hamiltonian (30) is quantized by imposing the canonical commutation relations $\left[\phi_{q, j}, n_{k}\right]=i \delta_{j k},\left[\phi_{1}, n\right]=i$. For later convenience, the oscillator is described in terms of the ladder operators

$$
\phi_{1}=\left(\frac{E_{C k}}{4 E_{L k}}\right)^{1 / 4}\left(a+a^{\dagger}\right), \quad n=i\left(\frac{E_{L k}}{4 E_{C k}}\right)^{1 / 4}\left(a^{\dagger}-a\right)
$$

with $\left[a, a^{\dagger}\right]=1$. The quantized oscillator Hamiltonian then reads

$$
H_{o s c}=\hbar \omega_{k}\left(\frac{1}{2}+a^{\dagger} a\right)
$$

with $\omega_{k}$ given by Eq. (16).

The Coulomb blockade effect in the SCB is taken into account by considering the periodicity of the SCB potential and imposing $2 \pi$-periodic boundary conditions on the wave function with respect to the phase $\phi_{q, j}$. The result is charge quantization on the island. Assuming the charge regime $E_{C} \gg E_{J}$ for the SCB and keeping the system at low temperature $\left(k_{B} T \ll E_{C}\right)$ and close to the charge degeneracy point $n_{g}=1 / 2$ restricts the number of excess charges on the island to zero or one Cooper pair. This allows us to truncate the SCB Hilbert space to these two lowest charge states $|0\rangle$ and $|1\rangle$.

It is advantageous to operate at the qubit charge degeneracy point, where the decoherence effect is minimized, ${ }^{29,30}$ and allow only small departures $\delta n_{g, j}=1 / 2-n_{g, j}$ from this point during single-qubit operations. Considering this, we write the quantized qubit Hamiltonian in the qubit eigenbasis at the charge degeneracy point $[|g\rangle,|e\rangle]=[|0\rangle+|1\rangle,|0\rangle-|1\rangle]$,

$$
H_{j}=E_{C, j} \delta n_{g, j} \sigma_{x, j}-\frac{E_{J, j}}{2} \sigma_{z, j} .
$$

The interaction Eq. (26) is proportional to $\sin \phi_{q, j}$, which transforms into $\sigma_{y, j}$ during the quantization procedure. It will be helpful during the discussion of two-qubit operations to express the interaction through the raising (lowering) qubit operators $\sigma_{-}|e\rangle=|g\rangle, \sigma_{+}|g\rangle=|e\rangle$,

$$
\sigma_{y, j}=i\left(\sigma_{+, j}-\sigma_{-, j}\right) .
$$

Thus the quantized interaction Hamiltonian reads

$$
H_{i n t, j}=i \frac{\kappa_{j}}{2}\left(a+a^{\dagger}\right)\left(\sigma_{-, j}-\sigma_{+, j}\right)
$$

where the interaction energy $\kappa_{j}$ is determined by the coupling constant $\alpha_{j}$ in Eq. (28),

$$
\kappa_{j}=\alpha_{j} E_{J, j}\left(\frac{E_{C k}}{4 E_{L k}}\right)^{1 / 4} .
$$

Equations (35) and (36) were derived for the charge limit $E_{C} \gg E_{J}$. However, they remain valid qualitatively also in the charge-phase regime $E_{C} \sim E_{J}$, which is more advantageous from the point of view of decoherence, as is well established. ${ }^{29}$ In this regime, the lowest Bloch states of the SCB Hamiltonian rather than the charge states form the computational basis. This is fully consistent with the quantum capacitance readout method ${ }^{32}$ for our system, which realizes projective measurement on the qubit eigenbasis. Transformation of the SCB eigenbasis from the charge regime to the charge-phase regime with increasing ratio $E_{J} / E_{C}$ was analyzed in Ref. 33. Applying this analysis to the present case in the Appendix we find that the qubit-cavity coupling remains transversal acquiring the form in Eq. (A12), 


$$
H_{i n t, j}=\frac{a+a^{\dagger}}{2}\left(\widetilde{\kappa}_{j} \sigma_{+, j}+\widetilde{\kappa}_{j}^{*} \sigma_{-, j}\right),
$$

with the coupling constant $\widetilde{\kappa}_{j}$ differing from the coupling $\kappa_{j}$ of the charge regime in Eq. (36) by a complex numerical function $f\left(E_{J, j} / E_{C, j}\right) \sim 1, \widetilde{\kappa}_{j}=f\left(E_{J, j} / E_{C, j}\right) \kappa_{j}$. Such a modification does not change qualitatively the resonant qubit-cavity dynamics discussed in the next sections.

\section{B. Constraints}

To conclude our discussion of the qubit-cavity circuit, we summarize the imposed constraints on the circuit parameters required for a proper functioning of the circuit.

First, we required the phase regime for the SQUID and the cavity, implying $(2 e)^{2} /\left(2 C_{s}\right)=E_{C s} \ll \hbar \omega_{s} \ll E_{J s}(f)$ and $E_{C k} \ll \hbar \omega_{k} \ll E_{L k}$, respectively. The cavity capacitance in practice greatly exceeds, by several orders, the SQUID junction capacitances, $C_{k} \gg C_{s}$, while the cavity inductance must be comparable to the SQUID variable inductance, which is required by the dispersion equation (11), for $k d \sim 1$. Thus, the SQUID plasma frequency is typically much larger than the cavity frequency, $\omega_{s} \gg \omega_{k}$. The latter, in its turn, must be comparable to the qubit frequencies to provide the resonant coupling, $\omega_{k} \approx E_{J 1} / \hbar, E_{J 2} / \hbar$.

The qubit interaction with the cavity must not be too strong in order to provide sufficient off-resonance decoupling, $\kappa \sim\left(C_{c} / C_{\Sigma}\right) E_{J} \ll\left|E_{J, 2}-E_{J, 1}\right|$. On the other hand, it must exceed the variation of the level spacings in the cavity energy spectrum Eq. (24), $\kappa \gg E_{C k}$, caused by nonlinearity.

All these requirements can be collected in a chain of inequalities formulating the hierarchy of relevant circuit energies

$$
\begin{gathered}
E_{C k} \ll \kappa \ll\left|E_{J 1}-E_{J 2}\right| \leq \hbar \omega_{k} \sim E_{J}, \\
\hbar \omega_{k}, E_{C s} \ll \hbar \omega_{s} \ll E_{L k} \sim E_{J s} .
\end{gathered}
$$

There is an additional requirement imposed on the lower bound of the variation of the Josephson energy of the SQUID: the critical current through the SQUID should be much larger than the amplitude of the current fluctuations in the cavity. The latter is estimated for the zero-point fluctuations as $(\hbar / 2 e)\left(k d / L_{c a v}\right)\left(E_{C k} / E_{L k}\right)^{1 / 4}$, while the critical current of the SQUID is $(2 e / \hbar) E_{J S}$. Thus the requirement is equivalent, by virtue of Eq. (11), to the inequality $\tan (k d)>\left(E_{C k} / E_{L k}\right)^{1 / 4}$.

\section{TWO-QUBIT OPERATIONS}

A general way of performing two-qubit operations is to sequentially drive the cavity frequency in and out of resonance with the qubits, i.e., $\hbar \omega_{k}-E_{J, j}=\delta,|\delta| \ll \kappa_{j}$. The speed should be comparable to the scale of the qubit-cavity coupling frequency but small on the scale of the oscillator frequency, to prevent unwanted excitation of higher oscillator levels, $\partial_{t} \omega_{k} / \omega_{k} \sim \kappa / \hbar \ll \hbar \omega_{k}$. During the two-qubit operations, each qubit is parked at its charge degeneracy point, and an appropriate difference in the qubit energies,

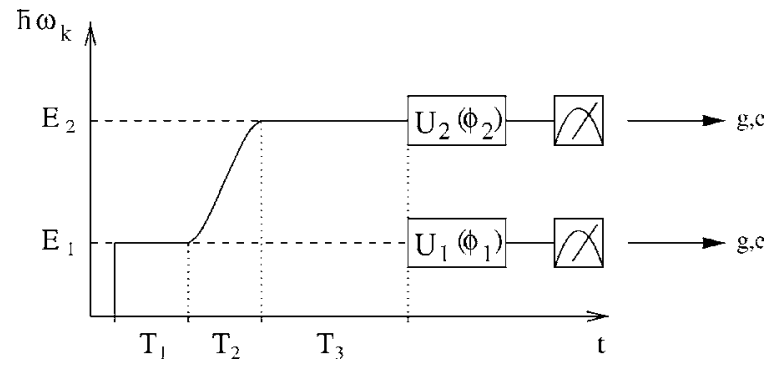

FIG. 4. Protocol for creating a Bell pair: The cavity frequency is sequentially swept through resonances with both qubits; at the first resonance the oscillator is entangled with qubit 1 , at the next resonance the oscillator swaps its state onto qubit 2 and ends up in the ground state. A Bell measurement is performed by applying Rabi pulses to noninteracting qubits, and projecting on the qubit eigenbasis $\{|g\rangle,|e\rangle\}$ by measuring quantum capacitance.

$\left|E_{J, 1}-E_{J, 2}\right| \gg \kappa$, prevents the oscillator from simultaneously interacting with both qubits.

Consider, as an example, qubit 1 in resonance with the oscillator. Discarding fast terms $\left(e^{ \pm i\left(\omega_{k}+E_{J, 1} / \hbar\right) t}\right.$ and $e^{ \pm i\left(\omega_{k} \pm E_{J, 2} / \hbar\right) t}$ ) in the interaction picture (with $H_{0}=H_{1}+H_{2}$ $+H_{\text {osc }}$ ), which average to zero on the time scale of the qubitoscillator interaction in the rotating wave approximation, the qubit-oscillator interaction term reads

$$
H_{\text {int }} \rightarrow i \frac{\kappa_{1}}{2}\left(a^{\dagger} \sigma_{-, 1}-a \sigma_{+, 1}\right) .
$$

The only nonzero interaction matrix elements, in the qubit-1-oscillator basis, are $\left\langle e, n\left|\sigma_{+} a\right| g, n+1\right\rangle$ $=\left\langle g, n+1\left|\sigma_{-} a^{\dagger}\right| e, n\right\rangle=\sqrt{n+1}$ between the levels which are close to being degenerate, $E_{n+1, g}-E_{n, e}=\delta$. The (truncated) Hamiltonian of this subspace reads

$$
H=-\frac{\delta}{2} \sigma_{z}+\frac{\kappa_{1}}{2} \sqrt{n+1} \sigma_{y} .
$$

While discussing the entangling operations, in the following we assume, to obtain analytical results, rectangular pulse shapes bringing the cavity in and out of exact resonance with the qubit $(\delta=0)$. The result of such an operation is given by the unitary matrix

$$
U(\theta)=\left(\begin{array}{cc}
\cos \theta & -\sin \theta \\
\sin \theta & \cos \theta
\end{array}\right), \quad \theta=\frac{\kappa_{1}}{2} \sqrt{n+1} T,
$$

where $T$ is the pulse duration. A slight detuning within the allowed interval $\delta \ll \kappa_{j}$ and smoother pulse shape will not qualitatively alter the protocols.

\section{A. Bell measurement protocol}

Maximally entangled two-qubit states (Bell states) are constructed from an initial state with one excitation, say $|e g 0\rangle$, and partly moving this excitation to the other qubit by sequentially sweeping the oscillator through resonance with both qubits. ${ }^{20}$ The oscillator starts and ends in the ground state. The first pulse brings the cavity to resonance with the excited qubit $1, \hbar \omega_{k}=E_{1}$ (see Fig. 4) during a time satisfying 
the relation, $\kappa_{1} T_{1}=\hbar \pi / 2(\pi / 2$ pulse $)$. Then the cavity, which has become entangled with qubit 1 ,

$$
|e g 0\rangle \rightarrow \frac{1}{\sqrt{2}}(|\operatorname{eg} 0\rangle+|g g 1\rangle),
$$

is driven toward resonance with qubit 2. The accumulated phase during the free evolution $\left(\hbar \omega_{k} \neq E_{1}, E_{2}\right.$ for a time $\left.T_{2}\right)$ is $\varphi_{2}$,

$$
\frac{1}{\sqrt{2}}\left(|\operatorname{eg} 0\rangle+e^{i \varphi_{2}}|g g 1\rangle\right), \quad \varphi_{2}=\int_{0}^{T_{2}}\left(\frac{E_{1}}{\hbar}-\omega_{k}(t)\right) d t .
$$

After the oscillator has reached resonance with qubit 2, $\hbar \omega_{k}=E_{2}$, it stays in resonance during the time, $\kappa_{2} T_{3}=\hbar \pi(\pi$ pulse). The system evolves to the state

$$
\frac{1}{\sqrt{2}}\left(|e g 0\rangle-e^{i\left(E_{1}-E_{2}\right) T_{3} / \hbar} e^{i \varphi_{2}}|g e 0\rangle\right)
$$

Choosing the time $T_{2}$ of free evolution such that the accumulated phase $\varphi_{2}$ satisfies the equation $\exp \left[i\left(E_{1}-E_{2}\right) T_{3} / \hbar\right.$ $\left.+i \varphi_{2}\right]=\mp 1$, the two-qubit system ends up in one of the Bell states

$$
\left|\Psi_{ \pm}\right\rangle=\frac{1}{\sqrt{2}}(|e g\rangle \pm|g e\rangle)|0\rangle
$$

Note that the cavity has returned to its ground state.

To perform a test of the nonclassical statistical properties of the Bell state Eq. (43), it is convenient to implement a protocol similar to the one of Ref. 31 for measuring the $\mathrm{CHSH}$ inequality. ${ }^{23}$ The protocol consists of two independent rotations of the uncoupled qubits by means of applying Rabi pulses, and then measuring the quantum capacitances of both $\mathrm{SCB} .^{32}$ The latter realizes a projective measurement on the qubit eigenstates $\left|g_{j}\right\rangle,\left|e_{j}\right\rangle$.

The resonant $\pi / 2$ pulse applied to the gate of the $j$ th qubit,

$$
\delta n_{g, j}(t)=\delta n_{g, j}^{0} \cos \left(E_{J, j} t\right),
$$

during the time $T_{a}$ such that $T_{a}=\hbar \pi /\left(2 E_{C} \delta n_{g, j}^{0}\right)$ produces a unitary transformation

$$
U_{j}\left(\phi_{j}\right)=\left(\begin{array}{cc}
1 & -i e^{-i \phi_{j}} \\
-i e^{i \phi_{j}} & 1
\end{array}\right), \quad \phi_{j, a}=E_{J, j} T_{a} / \hbar .
$$

By applying such pulses to both qubits, one gets the state

$$
\Psi_{ \pm}\left(\phi_{1}, \phi_{2}\right)=U_{1}\left(\phi_{1}\right) U_{2}\left(\phi_{2}\right) \Psi_{ \pm} .
$$

After the measurement, the qubits will be found either in similar states (both qubits in the ground $|g g\rangle$ or excited $|e e\rangle$ states), or in different states $(|g e\rangle$ or $|e g\rangle)$. The corresponding correlation function reads

$$
q_{ \pm}\left(\phi_{1}, \phi_{2}\right)=\left\langle\Psi_{ \pm}\left(\phi_{1}, \phi_{2}\right)\left|\sigma_{z, 1} \sigma_{z, 2}\right| \Psi_{ \pm}\left(\phi_{1}, \phi_{2}\right)\right\rangle .
$$

By repeating the procedure with a $\pi / 2$ pulse of slightly different duration $T_{b}$, with $T_{b}-T_{a} \sim \hbar / E_{J, j} \ll T_{a}$, we obtain the four correlation functions

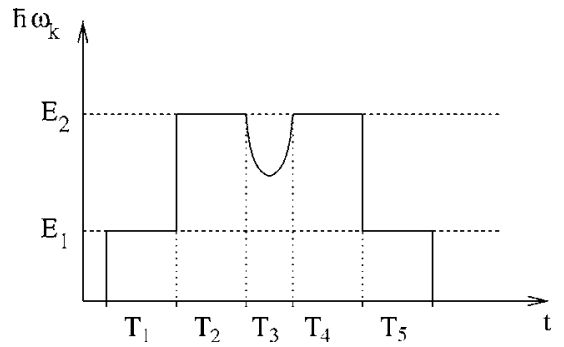

FIG. 5. Pulse sequence producing (trivial) diagonal gate: during time $T_{1}$, qubit 1 swaps its state onto the oscillator, then the oscillator interacts with qubit 2 before swapping its state back onto qubit 1 ; free evolution during time $T_{3}$ is added to annihilate the twophoton state in the cavity.

$$
q\left(\phi_{1 a}, \phi_{2 a}\right), \quad q\left(\phi_{1 b}, \phi_{2 a}\right), \quad q\left(\phi_{1 a}, \phi_{2 b}\right), \quad q\left(\phi_{1 b}, \phi_{2 b}\right) .
$$

According to the analysis in Ref. 23, the quantity

$$
B=\left|q\left(\phi_{1 a}, \phi_{2 a}\right)-q\left(\phi_{1 b}, \phi_{2 a}\right)\right|+\left|q\left(\phi_{1 a}, \phi_{2 b}\right)+q\left(\phi_{1 b}, \phi_{2 b}\right)\right|
$$

has an upper bound for classical statistics, $B \leq 2$. For the state in Eq. (43), however, we have

$$
q_{ \pm}\left(\phi_{1}, \phi_{2}\right)= \pm \cos \left(\phi_{1}-\phi_{2}\right),
$$

and the upper bound for $B$ becomes $B \leq 2 \sqrt{2}$, the equality being achieved for

$$
\phi_{1 a}=-\phi_{2 a}=\frac{3 \pi}{8}, \quad \phi_{1 b}=-\phi_{2 b}=-\frac{\pi}{8} .
$$

\section{B. Two-qubit control-phase gate}

In this section we modify the Bell state construction to implementing a control-phase two-qubit gate. This gate has the diagonal form $|\alpha \beta 0\rangle \rightarrow \exp \left(i \phi_{\alpha \beta}\right)|\alpha \beta 0\rangle \quad\left(\phi_{00}=\phi_{01}=\phi_{10}\right.$ $\left.=0, \phi_{11}=\pi\right)$, and it is equivalent to the controlled-NOT (CNOT) gate (up to local rotations). To generate such a diagonal gate, we adopt the following strategy: First tune the oscillator through resonance with both qubits performing $\pi$-pulse swaps in every step, and then reverse the sequence, as shown in Fig. 5. With an even number of swaps at every level, clearly the resulting gate will be diagonal.

Such a strategy would indeed produce the CPHASE gate provided the qubit-cavity coupling constant in Eq. (40) does not depend on the number of photons. Indeed, applying the rectangular $\pi$ pulse to pass the first resonance $\kappa_{1} T_{1}=\pi \hbar$ and then the $\pi$ pulse for the second resonance $\kappa_{2} T_{2}=\pi \hbar$, and then reversing the pulse sequence $\kappa_{2} T_{4}=\pi \hbar$ and $\kappa_{1} T_{5}=\pi \hbar$ (see Fig. 5), we induce the following transitions: 


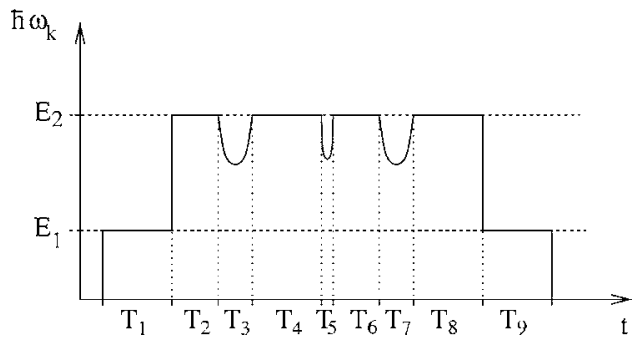

FIG. 6. Correct pulse sequence for performing a control-phase gate: time intervals $T_{1}, T_{4}, T_{8}$, and $T_{9}$ are single-photon $\pi$ pulses, whereas $T_{2}$ and $T_{6}$ are two-photon $\pi$ pulses; free evolution during times $T_{3}, T_{5}$, and $T_{7}$ is added to annihilate excited photon states, and create a nontrivial phase shift.

$$
\begin{aligned}
& |g g 0\rangle \rightarrow|g g 0\rangle \stackrel{2}{\rightarrow}|\operatorname{gg} 0\rangle \stackrel{4}{\rightarrow}|\operatorname{gg} 0\rangle \stackrel{5}{\rightarrow}+|g g 0\rangle, \\
& |g e 0\rangle \rightarrow|g e 0\rangle \rightarrow|g g 1\rangle \rightarrow-|g e 0\rangle \rightarrow-|g e 0\rangle, \\
& |e g 0\rangle \rightarrow|g g 1\rangle \rightarrow-|g e 0\rangle \rightarrow-|g g 1\rangle \rightarrow+|e g 0\rangle, \\
& |e e 0\rangle \rightarrow|g e 1\rangle \rightarrow|g g 2\rangle \rightarrow-|g e 1\rangle \rightarrow+|e e 0\rangle,
\end{aligned}
$$

which generate the CPHASE gate. The problem is, however, that it is not possible to make the swaps for all the states at the second resonance, e.g., $|g e 0\rangle \rightarrow|g g 1\rangle$, and $|g e 1\rangle \rightarrow|g g 2\rangle$, with the same pulse because of different values of the coupling constants, $\kappa_{2}$ and $\kappa_{2} \sqrt{2}$, respectively. Thus the $\pi$ pulse for the first transition will necessarily produce a state superposition for the second transition, and vice versa. There is a possibility to annihilate this superposition by inserting an interference loop in the pulse sequence (see Fig. 5), namely, by departing from the resonance for a while to accumulate an appropriate phase shift during the free evolution; the required time $T_{3}$ for such an excursion is given by the equation

$$
\theta_{3}=\int_{0}^{T_{3}}\left(\frac{E_{2}}{\hbar}-\omega_{k}(t)\right) d t=\pi \quad(\bmod 2 \pi)
$$

However, it is easy to check that such a protocol will generate a nonentangling, trivial gate.

It turns out that by adding two more swap segments with interference loops to the previous protocol it is possible to obtain a sufficient amount of free parameters to annihilate the state superpositions, and to obtain an entangling gate. ${ }^{24}$ The pulse sequence is shown in Fig. 6, and it consists, at the second resonance, of the two $\pi$ pulses producing swaps to the single-photon states $\kappa_{2} T_{4}=\kappa_{2} T_{8}=\hbar \pi$, as well as the two $\pi$ pulses producing swaps to the two-photon states $\sqrt{2} \kappa_{2} T_{2}$ $=\sqrt{2} \kappa_{2} T_{6}=\hbar \pi$. The first and the third interference loops are included in the pulse sequence to annihilate the state superpositions, the corresponding phase shifts satisfying the relations

$$
\theta_{3}-\theta_{5}=\pi, \quad \theta_{5}-\theta_{7}=\pi \quad(\bmod 2 \pi),
$$

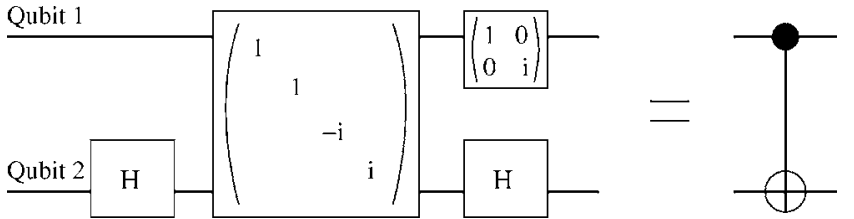

FIG. 7. Gate circuit for constructing a CNOT gate using the control-phase gate: a $z$-axis rotation is applied to qubit 1, and Hadamard gates $\mathrm{H}$ are applied to the second qubit.

$$
\theta_{n}=\int_{0}^{T_{n}}\left(\frac{E_{2}}{\hbar}-\omega_{k}(t)\right) d t
$$

The middle loop is required for generating a nontrivial gate. Specifically, the pulse sequence $\left(T_{2}-T_{8}\right)$ produces on the states $|g e 0\rangle$ and $|g g 1\rangle$ the following gate operation:

$$
U\left(\frac{\pi}{2}\right) S_{7} U\left(\frac{\pi}{2 \sqrt{2}}\right) S_{5} U\left(\frac{\pi}{2}\right) S_{3} U\left(\frac{\pi}{2 \sqrt{2}}\right)=e^{-i \theta_{5}}\left(\begin{array}{cc}
1 & 0 \\
0 & e^{-i \theta_{5}}
\end{array}\right),
$$

where $S_{n}=\operatorname{diag}\left[e^{-i \theta_{n}}, 1\right]$. For the levels $|g e 1\rangle$ and $|g g 2\rangle$, the sequence produces the gate operation

$$
U\left(\frac{\pi}{\sqrt{2}}\right) S_{7} U\left(\frac{\pi}{2}\right) S_{5} U\left(\frac{\pi}{\sqrt{2}}\right) S_{3} U\left(\frac{\pi}{2}\right)=e^{-i \theta_{5}}\left(\begin{array}{cc}
1 & 0 \\
0 & e^{-i \theta_{5}}
\end{array}\right) .
$$

Incorporating the modified gate operation into the sequence Eq. (52), we end up with the state evolution corresponding to a diagonal gate operation:

$$
\begin{aligned}
& |g g 0\rangle \stackrel{1}{\rightarrow}|g g 0\rangle \stackrel{2-8}{\rightarrow} \quad|g g 0\rangle \stackrel{9}{\rightarrow}+|g g 0\rangle, \\
& |g e 0\rangle \rightarrow|g e 0\rangle \rightarrow e^{-i \theta_{5}}|g e 0\rangle \rightarrow e^{-i \theta_{5}}|g e 0\rangle, \\
& |e g 0\rangle \rightarrow|g g 1\rangle \rightarrow e^{-i 2 \theta_{5}}|g g 1\rangle \rightarrow-e^{-i 2 \theta_{5}}|e g 0\rangle, \\
& |e e 0\rangle \rightarrow|g e 1\rangle \rightarrow e^{-i \theta_{5}}|g e 1\rangle \rightarrow-e^{-i \theta_{5}}|e e 0\rangle .
\end{aligned}
$$

The overall protocol produces the universal CPHASE gate (up to a common phase and single-qubit rotations),

$$
\begin{aligned}
& |g g 0\rangle \\
& |g e 0\rangle \\
& |e g 0\rangle \\
& |e e 0\rangle
\end{aligned} \rightarrow\left[\begin{array}{lllll}
1 & & & \\
& 1 & & \\
& & e^{-i \theta_{5}} & \\
& & & e^{i \theta_{5}}
\end{array}\right] \begin{aligned}
& |g g 0\rangle \\
& |g e 0\rangle \\
& |e g 0\rangle \\
& |e e 0\rangle
\end{aligned} .
$$

For a specific choice of the angle $\theta_{5}=\pi / 2(\bmod 2 \pi)$, the gate in Eq. (56) can be further transformed to a CNOT gate using Hadamard rotations on qubit 2 as shown in Fig. 7.

\section{DISCUSSION}

The protocol for the CPHASE gate considered in the previous section is much faster than the one suggested for a similar qubit-oscillator system and based on the dispersive qubitoscillator coupling ${ }^{20}$ [by the ratio of the coupling frequency to the deviation from the exact resonance (detuning)]. On the other hand, the CPHASE gate protocol discussed in this paper 
is more complex and relatively slower than the protocol for direct qubit-qubit $z z$ coupling considered in Refs. 12 and 33: the duration of the gate operation in the latter case is $h / 8$ in the units of inverse coupling energy, while it is $2.7 h$ for the protocol presented in Fig. 6. This illustrates the advantage of longitudinal, $z z$ coupling (in the qubit eigenbasis), which is achieved for the charge qubits biased at the charge degeneracy point by current-current coupling. ${ }^{12,33}$ More common for charge qubits is the capacitive coupling, however there the situation is different: this coupling has $x x$ symmetry at the charge degeneracy point, and because of inevitable difference in the qubit frequencies, the gate operation takes much longer time, prolonged by the ratio between the qubits frequency asymmetry and the coupling frequency. Recent suggestions to employ dynamic control methods to effectively bring the qubits into resonance ${ }^{34,35}$ can speed up the gate operation. For these protocols, the gate duration is $\sim h$ in units of direct coupling energy, which is longer than in the case of $z z$ coupling, but somewhat shorter than in our case. However, the protocol considered in this paper might be made faster by using pulse shaping.

A specific feature of the present protocol is that there is always at least one qubit staying off resonance: one qubit stays off resonance during manipulations with the other qubit, and also the second qubit undergoes off-resonance excursions during the manipulation. During these periods, free evolution of the qubits is assumed; however, a finite offresonant qubit-cavity coupling violates this assumption and eventually negatively affects the fidelity of the gate. Therefore it is particularly important for this protocol to provide a weak off-resonance interaction. The latter is perturbatively estimated as $\sim \kappa^{2} / E_{J}$, which is by a factor $\sim C_{c} / C_{\Sigma}$ smaller than the resonant interaction. In practice this factor would not exceed $1 / 100$ (coupling frequency $\sim 80 \mathrm{MHz}$ for qubit frequency $>5 \mathrm{GHz}$ ), which would provide a fidelity of the gate not worse than $1 \%$.

To estimate the maximum number of qubits the circuit is able to accommodate, we assume reduced coupling strength $\kappa / h \sim 10 \mathrm{MHz}$ (which would still allow up to ten two-qubit operations during optimistic $1 \mu$ s decoherence time). Assuming the same gate fidelity, $1 \%$, and given the fact that the maximum qubit frequency is in the range of $E_{J} / h$ $\sim 10 \mathrm{GHz}$, we find that the number of qubits cannot be more than ten qubits. Thus we conclude that the resonant method of selective qubit addressing considered in the present paper allows one to implement a small quantum processor suitable for testing the simplest quantum algorithms, although it has limited potential for larger-scale applications.

\section{ACKNOWLEDGMENTS}

We thank Chris Wilson, Per Delsing, Martin Sandberg, Göran Johansson, and Lars Tornberg for helpful discussions. Support by the European Union under Contracts No. IST-3015708-IP EuroSQIP and No. IST-SQUBIT-2, by SSFNANODEV, and by the Swedish Research Council is gratefully acknowledged.

\section{APPENDIX: CHARGE-PHASE REGIME}

In this appendix we derive Eq. (37) for the qubit-cavity interaction in the charge-phase regime.
The starting point is the SCB Hamiltonian in Eq. (31) at the degeneracy point $n_{g}=1 / 2$, written in the charge basis $|n\rangle$,

$$
H=\sum_{n=-\infty}^{\infty}\left[E_{C}(n-1 / 2)^{2}|n\rangle\langle n|-\left(E_{J} / 2\right)(|n+1\rangle\langle n|+| n-1\rangle\langle n|)\right] .
$$

We split the complete set of the charge eigenstates, $-\infty<n<\infty$, in the positive and negative charge subsets labeled with $\sigma=\uparrow, \downarrow$, and $m=\ldots, 2,1$ such that

$$
m= \begin{cases}n, & n>0 \\ 1-n, & n \leq 0\end{cases}
$$

In the basis $|m, \sigma\rangle$, the Hamiltonian $H$ acquires the form

$$
H=\left[\begin{array}{ll}
A & B \\
B & A
\end{array}\right],
$$

where $A$ is the tridiagonal matrix

$$
A=\frac{E_{C}}{4}\left[\begin{array}{cccc}
\ddots & \ddots & \ddots & \\
& -p & 9 & -p \\
& & -p & 1
\end{array}\right], \quad p=\frac{2 E_{J}}{E_{C}},
$$

and matrix $B$ contains only a single element,

$$
B=-\frac{E_{J}}{2}\left[\begin{array}{lll}
\ddots & & \vdots \\
& 0 & 0 \\
\ldots & 0 & 1
\end{array}\right] \text {. }
$$

Similarly, the interaction Hamiltonian corresponding to Eqs. (26), (28), and (36),

$$
H_{\text {int }}=\frac{i \kappa}{2}\left(a+a^{\dagger}\right) \sum_{n=-\infty}^{\infty}(|n+1\rangle\langle n|-| n-1\rangle\langle n|),
$$

takes the form in the $(m, \sigma)$ representation,

$$
H_{\text {int }}=\left(a+a^{\dagger}\right)\left[\begin{array}{cc}
C & i D \\
-i D & -C
\end{array}\right],
$$

where

$$
C=\frac{\kappa}{2}\left[\begin{array}{cccc}
\ddots & \ddots & \ddots & \\
& -i & 0 & i \\
& & -i & 0
\end{array}\right], \quad D=\frac{\kappa}{2}\left[\begin{array}{ccc}
\ddots & & \vdots \\
& 0 & 0 \\
\ldots & 0 & 1
\end{array}\right] .
$$

A Hadamard rotation $\mathrm{H}$ in $\sigma$ space,

$$
\mathrm{H}=\frac{1}{\sqrt{2}}\left(\sigma_{z}+\sigma_{x}\right)
$$

takes the basis $|m \uparrow\rangle,|m \downarrow\rangle$ to $|m \pm\rangle=(1 / \sqrt{2})(|m \uparrow\rangle \pm|m \downarrow\rangle)$, and transforms the matrices in Eqs. (A3) and (A7) to the form

$$
H=A \mathbf{1}+B \sigma_{z}, \quad H_{\text {int }}=\left(a+a^{\dagger}\right)\left(C \sigma_{x}+D \sigma_{y}\right) .
$$

The eigenstates of the SCB Hamiltonian, the Bloch states, which consist of superpositions of many charge states, can be 
found by independent unitary rotations in the $\sigma=+$ and $\sigma=-$ subspaces due to the block-diagonal form of the matrix $H$ in Eq. (A10).

The qubit states are chosen as the two lowest-energy Bloch states. The corresponding energy eigenvalues occupythe bottom right corners of the diagonalized matrices $\left[U^{\dagger}(A+B) U\right]_{11}$ and $\left[V^{\dagger}(A-B) V\right]_{11}$, where $U(p)$ and $V(p)$ are appropriate unitary matrices. Indeed, this is obviously true for the charge regime $(p \ll 1)$, when these matrix elements correspond to superpositions of the $n=0$ and $n=1$ charge states. When the Josephson energy increases, which corresponds to increasing potential energy in the Bloch Hamiltonian $(p \sim 1)$, the Bloch energies change without crossing, ${ }^{36}$ and therefore the bottom right corner matrix elements remain the lowest energy eigenvalues.
In the qubit subspace, the interaction Hamiltonian in Eq. (A10) takes the form

$$
H_{\text {int }}=\left(a+a^{\dagger}\right)\left[\begin{array}{cc}
0 & {\left[U^{\dagger}(C-i D) V\right]_{11}} \\
{\left[V^{\dagger}(C+i D) U\right]_{11}} & 0
\end{array}\right] .
$$

Denoting the matrix element of the Hermitian matrix in Eq. (A11) as $\widetilde{\kappa} / 2=f(p) \kappa / 2$, where $f(p)$ is a complex numerical coefficient, we arrive at the qubit-cavity interaction in the form

$$
H_{\text {int }}=\frac{a+a^{\dagger}}{2}\left(\widetilde{\kappa} \sigma_{+}+\widetilde{\kappa}^{*} \sigma_{-}\right)
$$

${ }^{1}$ Yu. A. Pashkin, T. Yamamoto, O. Astafiev, Y. Nakamura, D. V. Averin, and J. S. Tsai, Nature (London) 421, 823 (2003).

${ }^{2}$ T. Yamamoto, Yu. A. Pashkin, O. Astafiev, Y. Nakamura, and J. S. Tsai, Nature (London) 425, 941 (2003).

${ }^{3}$ J. B. Majer, F. G. Paauw, A. C. J. ter Haar, C. J. P. M. Harmans, and J. E. Mooij, Phys. Rev. Lett. 94, 090501 (2005).

${ }^{4}$ A. C. J. ter Haar, Ph.D. thesis, Delft University, 2005.

${ }^{5}$ A. Izmalkov, M. Grajcar, E. Il'ichev, Th. Wagner, H. G. Meyer, A. Yu. Smirnov, M. H. S. Amin, A. Maassen van den Brink, and A. M. Zagoskin, Phys. Rev. Lett. 93, 037003 (2004).

${ }^{6}$ A. J. Berkley, H. Xu, R. C. Ramos, M. A. Gubrud, F. W. Strauch, P. R. Johnson, J. R. Anderson, A. J. Dragt, C. J. Lobb, and F. C. Wellstood, Science 300, 1548 (2003).

${ }^{7}$ R. McDermott, R. W. Simmonds, M. Steffen, K. B. Cooper, K. Cicak, K. D. Osborn, S. Oh, D. P. Pappas, and J. M. Martinis, Science 307, 1299 (2005).

${ }^{8}$ G. Wendin and V. Shumeiko, in Handbook of Theoretical and Computational Nanotechnology, edited by M. Rieth and W. Schommers (ASP, Los Angeles, 2006), Vol. 3, pp. 223-309.

${ }^{9}$ D. V. Averin and C. Bruder, Phys. Rev. Lett. 91, 057003 (2003).

${ }^{10}$ Yu. Makhlin, G. Schön, and A. Shnirman, Rev. Mod. Phys. 73, 357 (2001).

${ }^{11}$ J. Q. You and F. Nori, Phys. Rev. B 68, 064509 (2003).

${ }^{12}$ J. Lantz, M. Wallquist, V. S. Shumeiko, and G. Wendin, Phys. Rev. B 70, 140507(R) (2004).

${ }^{13}$ A. Lyakhov and C. Bruder, New J. Phys. 7, 181 (2005).

${ }^{14}$ A. O. Niskanen, Y. Nakamura, and J.-S. Tsai, Phys. Rev. B 73, 094506 (2006).

15 J. I. Cirac, R. Blatt, A. S. Parkins, and P. Zoller, Phys. Rev. Lett. 70, 762 (1993); J. I. Cirac and P. Zoller, ibid. 74, 4091 (1995).

${ }^{16}$ C. Monroe, D. M. Meekhof, B. E. King, W. M. Itano, and D. J. Wineland, Phys. Rev. Lett. 75, 4714 (1995).

${ }^{17}$ A. Wallraff, D. I. Schuster, A. Blais, L. Frunzio, R.-S. Huang, J. Majer, S. Kumar, S. M. Girvin, and R. J. Schoelkopf, Nature (London) 431, 162 (2004).

${ }^{18}$ I. Chiorescu, P. Bertet, K. Semba, Y. Nakamura, C. J. P. M. Harmans, and J. E. Mooij, Nature (London) 431, 159 (2004).

${ }^{19}$ J. Johansson, S. Saito, T. Meno, H. Nakano, M. Ueda, K. Semba, and H. Takayanagi, Phys. Rev. Lett. 96, 127006 (2006).
${ }^{20}$ F. Plastina and G. Falci, Phys. Rev. B 67, 224514 (2003).

${ }^{21}$ A. Blais, A. Maassen van den Brink, and A. M. Zagoskin, Phys. Rev. Lett. 90, 127901 (2003).

${ }^{22}$ A. Blais, R.-S. Huang, A. Wallraff, S. M. Girvin, and R. J. Schoelkopf, Phys. Rev. A 69, 062320 (2004).

${ }^{23}$ J. F. Clauser, M. A. Horne, A. Shimony, and R. A. Holt, Phys. Rev. Lett. 23, 880 (1969).

${ }^{24}$ F. Schmidt-Kaler, H. Häffner, M. Riebe, S. Gulde, G. P. T. Lancaster, T. Deuschle, C. Becher, C. F. Roos, J. Eschner, and R. Blatt, Nature (London) 422, 408 (2003).

${ }^{25}$ B. Yurke and J. S. Denker, Phys. Rev. A 29, 1419 (1984).

${ }^{26}$ M. Devoret, in Quantum Entanglement and Information Processing, edited by D. Estève, J.-M. Raimond, and J. Dalibard, Proceedings of the Les Houches Summer School of Theoretical Physics, LXIII, 1995 (Elsevier, Amsterdam, 2004).

${ }^{27}$ A. Shnirman, G. Schön, and Z. Hermon, Phys. Rev. Lett. 79, 2371 (1997).

${ }^{28}$ During gate voltage operations, the additional term $i U^{\dagger} \partial_{t} U$ becomes relevant, shifting the oscillator to $E_{L k}\left[\phi_{1}+\dot{n}_{0} /\left(2 E_{L k}\right)\right]^{2}$. The shift is small compared to the gate voltage speed $\dot{n}_{g, i}$ because $\alpha \ll 1$.

${ }^{29}$ D. Vion, A. Cottet, A. Aassime, P. Joyez, H. Pothier, C. Urbina, D. Esteve, and M. H. Devoret, Science 296, 886 (2002).

${ }^{30}$ T. Duty, D. Gunnarsson, K. Bladh, and P. Delsing, Phys. Rev. B 69, 140503(R) (2004).

${ }^{31}$ M. A. Rowe, D. Kielpinski, V. Meyer, C. A. Sackett, W. M. Itano, C. Monroe, and D. J. Wineland, Nature (London) 409, 791 (2003).

${ }^{32}$ G. Johansson, L. Tornberg, V. S. Shumeiko, and G. Wendin, J. Phys.: Condens. Matter 18, 901 (2006); G. Johansson, L. Tornberg, and C. Wilson, Phys. Rev. B 74, 100504(R) (2006).

${ }^{33}$ M. Wallquist, J. Lantz, V. S. Shumeiko, and G. Wendin, New J. Phys. 7, 178 (2005).

${ }^{34}$ C. Rigetti, A. Blais, and M. Devoret, Phys. Rev. Lett. 94, 240502 (2005).

${ }^{35}$ P. Bertet, C. J. P. M. Harmans, and J. E. Mooij, Phys. Rev. B 73, 064512 (2006).

${ }^{36}$ C. M. Bender and S. A. Orszag, Advanced Mathematical Methods for Scientists and Engineers (Springer-Verlag, New York, 1999). 